\title{
Correlated-Gaussian calculations of the ground and low-lying excited states of the boron atom
}

\author{
Sergiy Bubin ${ }^{1}$ and Ludwik Adamowicz ${ }^{2,3}$ \\ ${ }^{1}$ Department of Physics and Astronomy, Vanderbilt University, Nashville, Tennessee 37235, USA \\ ${ }^{2}$ Department of Chemistry and Biochemistry, University of Arizona, Tucson, Arizona 85721, USA \\ ${ }^{3}$ Department of Physics, University of Arizona, Tucson, Arizona 85721, USA
}

(Received 20 December 2010; published 16 February 2011)

\begin{abstract}
Benchmark variational calculations of the four lowest ${ }^{2} P$ and ${ }^{2} S$ states of the boron atom (including the ground state) have been performed. The wave functions of the states have been expanded in terms of all-particle explicitly correlated Gaussian basis functions and the finite mass of the nucleus has been explicitly accounted for.Variational upper bounds for the nonrelativistic finite- and infinite-nuclear-mass energies of all considered states have been obtained with the relative convergence of the order of $10^{-7}-10^{-8}$. Expectation values of the powers of the inter-particle distances and Dirac $\delta$ functions depending on those distances have also been computed. These calculations provide reference values that can be used to test other high-level quantum chemistry methods.
\end{abstract}

DOI: 10.1103/PhysRevA.83.022505

PACS number(s): 31.15.ac, 31.15.V-, 31.15.xt

\section{INTRODUCTION}

There are several reasons for which finding accurate solutions of the Schrödinger equation for small atoms and molecules is important. Probably the most compelling one is to keep up with constantly improving experimental measurements. Much of the up-to-date atomic and molecular spectroscopic data have a resolution that exceeds $0.01-$ $0.001 \mathrm{~cm}^{-1}$ or even better. Unfortunately, theoretical techniques are usually unable to match such high accuracy values. With this, a good amount of potentially interesting physical phenomena remain inaccessible for quantitative (and often even qualitative) analysis and interpretation. For some atomic properties, the theory is orders of magnitude behind the experiment. It should be mentioned that the results obtained in high accuracy calculations of small few-electron systems when combined with high-resolution spectroscopic data make it possible to precisely determine such quantities as the fine-structure constant, electron-to-proton mass ratio, nuclear radii, quadrupole moments, etc.

Another important reason for performing accurate calculations on small atomic and molecular systems is that they can provide valuable reference data for the development and testing of less accurate quantum-chemical methods. A simple example is the total nonrelativistic energy of the system, which is one of the most commonly calculated quantities. This energy is difficult to determine very precisely based on purely experimental data because, even if very accurate measurements of the ionization potentials and/or the dissociation energies are available, the procedure to determine the energy requires knowledge of the binding energies of subsystems and the exact contribution of relativistic and QED effects. These latter quantities, while readily available from the calculations, are not directly obtained in the experiment.

However desirable very precise calculations on small atoms are, the difficulties associated with obtaining a very accurate solution of the Schrödinger equation are quite substantial. The main cause of these difficulties is rooted in the multidimensional nature of the wave function. The amount of the computational work necessary for such a task grows very fast with the number of particles in the system. Among the methods that are capable of effectively describing the inter-particle correlation effects, the class of explicitly correlated methods has proven to be particularly powerful. In this class of methods, the wave function of the system is represented in terms of basis functions that explicitly depend not only on the positions of the particles but also on the inter-particle distances. When this type of wave function is combined with the Rayleigh-Ritz variational scheme, it can describe either the ground state or an excited state of a small atom, a molecule, or any other quantum-mechanical system with an unmatched accuracy. In the case of the most studied three-particle atomic system, the helium atom, the accuracy of the best recent calculations has been truly impressive and exceeded 40 decimal figures in the total energy [1-3]. Such accuracy was possible due to the use of a basis set that, apart from other necessary components, also included logarithmic terms that correctly describe the behavior of the wave function at three-particle coalescence points.

Unfortunately, including basis functions that are capable of exactly representing all fine nuances of the exact wave function is only possible for three-particle systems. Including such functions in the calculations of larger systems leads to extremely complicated Hamiltonian and overlap matrix elements. For this reason, the next smallest atom, $\mathrm{Li}$, has been computed with "only" 12- to 13-digit accuracy [4-6] using wave functions expanded in terms of the Hylleraas-type basis functions. These basis functions do not have proper behavior at the three-particle coalescence points, but the wave function constructed with them can still satisfy the Kato cusp conditions [7]. The calculations with Hylleraas-type basis functions are currently limited to four-particle (or three-electron) atomic systems. The extension of the approach to larger atoms again faces difficulties with the analytic evaluation of the multidimensional integrals. The same is true in the case of yet another type of basis function, i.e., explicitly correlated exponential functions (also known as explicitly correlated Slater-type functions) [8-11].

The achieved precision in the calculations on atoms larger than $\mathrm{Li}$ drops even further. The accuracy in the calculations of the $\mathrm{Be}$ atom and other four-electron atomic systems had remained significantly lower than the accuracy of the available spectroscopic data until very recently, when a series of calculations matching the experimental error bars were 
reported [12-18]. All those calculations were performed using the explicitly correlated Gaussian functions (ECGFs). These functions have the advantage that the algorithms for the Hamiltonian matrix elements with them can be derived in the general form for any number of electrons, thus overcoming the difficulties associated with the use of the Hylleraas-type functions and of the explicitly correlated Slater-type functions.

In this paper, we show that, if sufficient computational effort is invested, the correlated Gaussians are capable of producing results for the ground and some lower-lying excited states of a five-electron atomic system, the boron atom, which almost match the accuracy achieved for the four-electron systems. As will be described in the next section, the key component of the variational energy minimization procedure that leads to such accurate results is the analytical energy gradient determined with respect to the Gaussian nonlinear parameters, which are optimized.

\section{METHOD}

The standard atomic quantum-mechanical calculations are usually performed with infinite nuclear mass, i.e., with assuming the Born-Oppenheimer (BO) approximation. However, in calculations where very high accuracy is desired, the energy needs to include the effect of the finite mass of nucleus. This can be done by using a Hamiltonian, which represents the internal state of the system, to explicitly include the dependency on the nuclear mass. Such a Hamiltonian, called here the internal Hamiltonian, is obtained from the laboratoryframe nonrelativistic Hamiltonian by rigorously separating out the center-of-mass motion. For an atom with $n$ electrons, this is done by a transformation from a laboratory coordinate frame, which describes the positions and the nucleus and the electrons of the atom, to a new set of coordinates comprising three Cartesian coordinates of the center of mass and $n$ internal Cartesian coordinates describing the positions of the electrons with respect to the nucleus. By separating out the center-ofmass motion, we obtain the following internal Hamiltonian (for details, see [19-22]):

$$
\begin{aligned}
\hat{H}= & -\frac{1}{2}\left(\sum_{i=1}^{n} \frac{1}{\mu_{i}} \nabla_{\mathbf{r}_{i}}^{2}+\sum_{\substack{i, j=1 \\
i \neq j}}^{n} \frac{1}{m_{0}} \nabla_{\mathbf{r}_{i}} \cdot \nabla_{\mathbf{r}_{j}}\right) \\
& +\sum_{i=1}^{n} \frac{q_{0} q_{i}}{r_{i}}+\sum_{i>j=1}^{n} \frac{q_{i} q_{j}}{r_{i j}}
\end{aligned}
$$

where $\mathbf{r}_{i}$ is the distance between the $i$ th electron and the nucleus, $m_{0}$ is the nucleus mass $\left[m_{0}\left({ }^{10} \mathrm{Be}\right)=18247.4689 m_{e}\right.$ and $m\left({ }^{11} \mathrm{Be}\right)=20063.7375 m_{e}$, where $m_{e}$ the electron mass], $q_{0}$ is its charge, $q_{i}$ are electron charges, and $\mu_{i}=m_{0} m_{i} /$ $\left(m_{0}+m_{i}\right)$ are electron reduced masses. The Hamiltonian (1) describes the motion of $n$ (pseudo)electrons, the masses of which are the reduced masses, in the central field of the nuclear charge. This motion is coupled through the Coulombic interactions between the electrons and the nucleus $\sum_{i=1}^{n} \frac{q_{0} q_{i}}{r_{i}}$ and through the interactions between the electrons $\sum_{i>j=1}^{n} \frac{q_{i} q_{j}}{r_{i j}}$, where $r_{i j}=\left|\mathbf{r}_{j}-\mathbf{r}_{i}\right|$, as well as through the mass polarization term $-\frac{1}{2} \sum_{\substack{i, j=1 \\ i \neq j}}^{n}\left(1 / m_{0}\right) \nabla_{\mathbf{r}_{i}} \cdot \nabla_{\mathbf{r}_{j}}$.

In the calculations of atomic states with only $s$ electrons (in the leading configuration), we use the following all-electron, spherical ECGFs:

$$
\phi_{k}\left(\mathbf{r}_{1}, \mathbf{r}_{2}, \ldots, \mathbf{r}_{n}\right)=\exp \left[-\mathbf{r}^{\prime}\left(A_{k} \otimes I_{3}\right) \mathbf{r}\right]
$$

where $\mathbf{r}$ is a vector formed by the $\mathbf{r}_{1}, \mathbf{r}_{2}, \ldots, \mathbf{r}_{n}$ vectors stacked on top of each other, $A_{k}$ is a $n \times n$ symmetric matrix, $I_{3}$ is a $3 \times 3$ identity matrix, $\otimes$ is the Kronecker product symbol, and the prime indicates the matrix or vector transpose. As the basis functions used in describing bound states must be square integrable, restrictions must be imposed on the $A_{k}$ matrices. Each $A_{k}$ matrix must be positive definite. Rather than imposing restrictions on the $A_{k}$ matrix elements, which can be quite costly to handle in the computational implementation, we use the following Cholesky factored form of $A_{k}: A_{k}=L_{k} L_{k}^{\prime}$, where $L_{k}$ is a lower triangular matrix. With this representation, $A_{k}$ is automatically positive definite for any values of the $L_{k}$ matrix elements ranging from $-\infty$ to $\infty$. Thus, the variational energy minimization with respect to the $L_{k}$ parameters can be carried out without any restrictions imposed on the optimized parameters. It should be noted that the $L_{k} L_{k}^{\prime}$ representation of $A_{k}$ matrix does not limit the flexibility of basis functions, because any symmetric positive matrix can be represented in a Cholesky factored form.

To describe states with $(n-1) s$ electrons and a single $p$ electron, we used the following ECGFs in the wave-function expansion:

$$
\phi_{k}\left(\mathbf{r}_{1}, \mathbf{r}_{2}, \ldots, \mathbf{r}_{n}\right)=z_{m_{k}} \exp \left[-\mathbf{r}^{\prime}\left(A_{k} \otimes I_{3}\right) \mathbf{r}\right] .
$$

Here $m_{k}$ is an integer that depends on $k$ with values ranging from 1 to $n . m_{k}$ is also subject to optimization.

Appropriate symmetry projections are used to make the wave function of the atom antisymmetric with respect to the permutation of the electron labels. In this paper, we use the spin-free formalism. In this formalism, the symmetry projections acting on the spatial parts of the wave function, i.e., the basis functions, can be represented using the Young projection operators $\hat{Y}$, which are linear combinations of permutational operators $\hat{P}_{\gamma}$. As the Hamiltonian is invariant with respect to all permutations of the electrons, in calculating the overlap and Hamiltonian matrix elements, the permutational operators can be applied to the ket (or the bra) only. More specifically, the ket basis functions in those matrix elements are operated on with the operator $\hat{Y}^{\dagger} \hat{Y}$ (the dagger stands for conjugate), where the $\hat{Y}$ operator is derived using the appropriate Young tableaux for the state under consideration (for details of the formalism see, for example, [23]). For the doublet states of boron, the Young operator can be chosen as $\hat{Y}=\left(\hat{1}+\hat{P}_{23}\right)\left(\hat{1}+\hat{P}_{45}\right)(\hat{1}-$ $\left.\hat{P}_{24}\right)\left(\hat{1}-\hat{P}_{26}-\hat{P}_{46}\right)\left(\hat{1}-\hat{P}_{35}\right)$, where the nucleus is labeled as particle 1 , and the electrons are labeled as particles $2, \ldots, 6, \hat{1}$ is the identity operator, and $\hat{P}_{i j}$ is the permutation operator of the spatial coordinates of the $i$ th and $j$ th electrons.

\section{RESULTS AND DISCUSSION}

The two lowest ${ }^{2} S$ and two lowest ${ }^{2} P$ states of boron are considered in these calculations. The lowest ${ }^{2} P$ state is the boron ground state. In Fig. 1, we use the experimental data taken from NIST Atomic Spectra Database [24] to show where those considered states are positioned in the boron spectrum. 


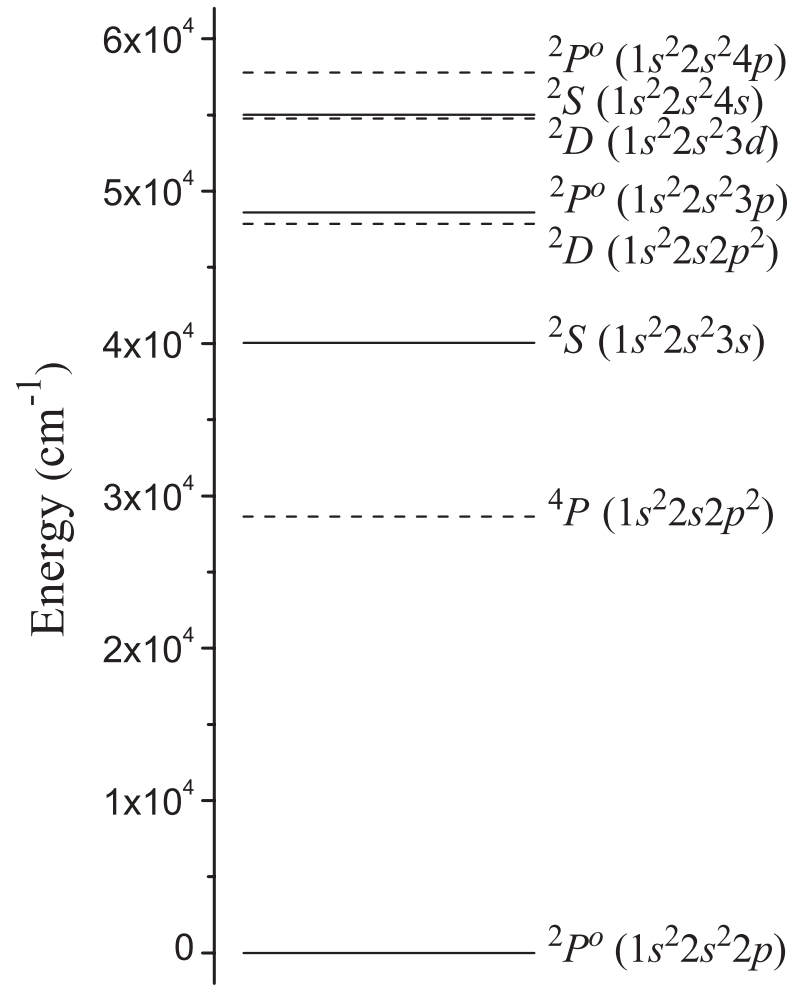

FIG. 1. The energy level diagram of the low-lying states of the boron atom. The four levels computed in this work are marked with the solid line.

The calculations have been carried out for ${ }^{10} \mathrm{~B}$ and ${ }^{11} \mathrm{~B}$ isotopes as well as for ${ }^{\infty} \mathrm{B}$; the latter one corresponds to the boron atom with an infinitely heavy nucleus. As the majority of quantum-chemical calculations are traditionally performed with an infinite nuclear mass, including the ${ }^{\infty} \mathrm{B}$ results here may provide a useful set of reference values for such calculations. The basis sets for different states have only been generated for the main ${ }^{11} \mathrm{~B}$ isotope and reused in the calculations of ${ }^{10} \mathrm{~B}$ and ${ }^{\infty} \mathrm{B}$. The reason for this time-saving simplification is due to the fact that, upon a small change of the wave function (the change remains small as long as the mass of the nucleus is significantly larger than that of electrons), it is sufficient to readjust only the linear coefficients of the wave function in order to recover the shift in the total energy. The simplification has virtually no effect on the accuracy of the calculation.

These calculations have been performed using the standard variational approach. In the variational energy minimization, the matrix elements of the $L_{k}$ matrices of the basis functions have been optimized. As mentioned, this was only done for the ${ }^{11} \mathrm{~B}$ isotope. For ${ }^{2} P$ states, the $m_{k}$ powers have been partially optimized. The optimization was carried out independently for each state and the basis set for each state has been grown incrementally from a small set of functions to the size of 5100 functions. Some preliminary results for ground state only and the basis size up to 2000 functions were reported in one of our previous works [25]. The initial selection of $L_{k}$ parameters (and $m_{k}$ for $P$ states) was done using a stochastic scheme similar to that described in other works [26-28]. After each function was added to the set, its $L_{k}$ parameters were optimized using a method that employs an analytical energy gradient determined with respect to the optimized parameters. After a subset of 10 functions were added to the basis, all functions in the set were reoptimized one by one using the gradientbased method. When the basis size reached 5000, we further increased the computational effort by changing the number of cycles performed after the addition of each new 10 basis functions from one to three. Also, starting from approximately the 5080 function basis, we switched from standard double precision (64-bit) to extended precision (80-bit) to improve the quality of the optimization, which is sensitive to the numerical accuracy of the computed eigenvalues and their derivatives.

The results of the calculations are presented in Table I. For the ${ }^{11} \mathrm{~B}$ isotope, the energies obtained with 1000,2000 , $3000,4000,5000$, and 5100 ECGFs are shown. This allows for an analysis of the energy convergence. We estimate that the energies for all considered states are converged to about $10^{-7}-10^{-8}$ relative to the respective energy values. For ${ }^{10} \mathrm{~B}$ and ${ }^{\infty} \mathrm{B}$, we only show the energies obtained with 5100 ECGFs. They are as well converged as the ${ }^{11} \mathrm{~B}$ energies.

Using the total energies of the states from Table I, we can determine the energies corresponding to transitions between states. These, in turn, can be directly compared with the experimental data taken from Ref. [24]. Such a comparison is presented in Table II. First let us examine the convergence of the calculated transition energies. This analysis can be done based on the transition energy values obtained for the ${ }^{11} \mathrm{~B}$ isotope for the different basis-set sizes. It shows that

TABLE I. The convergence of the total nonrelativistic energies (in Hartrees) with the basis size for the main isotope of boron atom ${ }^{11} \mathrm{~B}$. Energies obtained for ${ }^{10} \mathrm{~B}$ and ${ }^{\infty} \mathrm{B}$ with 5100 basis functions are also shown. The values in parentheses are estimates of the remaining uncertainty due to finite basis size used in the calculations.

\begin{tabular}{lccccc}
\hline \hline Isotope & Basis & ${ }^{2} P^{o}\left(1 s^{2} 2 s^{2} 2 p\right)$ & ${ }^{2} S\left(1 s^{2} 2 s^{2} 3 s\right)$ & ${ }^{2} P^{o}\left(1 s^{2} 2 s^{2} 3 p\right)$ & -24.40184139 \\
\hline${ }^{11} \mathrm{~B}$ & 1000 & -24.65249417 & -24.47010632 & -24.43097954 & -24.40192314 \\
& 2000 & -24.65259809 & -24.47013627 & -24.43107308 & -24.40193614 \\
& 3000 & -24.65261570 & -24.47014092 & -24.43108839 & -24.40193996 \\
& 4000 & -24.65262114 & -24.47014233 & -24.43109326 & -24.40194147 \\
& 5000 & -24.65262343 & -24.47014290 & -24.43109531 & $-24.40194183(150)$ \\
${ }^{10} \mathrm{~B}$ & $5100^{\mathrm{a}}$ & $-24.65262387(250)$ & $-24.47014316(50)$ & $-24.43109574(250)$ & $-24.40181783(150)$ \\
${ }^{\infty} \mathrm{B}$ & $5100^{\mathrm{a}}$ & $-24.65250024(250)$ & $-24.47001876(50)$ & $-24.43097175(250)$ & $-24.40318767(150)$ \\
\hline \hline
\end{tabular}

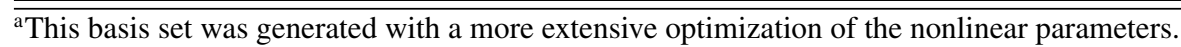


TABLE II. Nonrelativistic transition frequencies $\left(\right.$ in $^{-1}$ ) for different isotopes of B compared to the experimental values.

\begin{tabular}{|c|c|c|c|c|c|c|c|}
\hline Isotope & Basis & ${ }^{2} P^{o}(2 p) \rightarrow{ }^{2} S(3 s)$ & ${ }^{2} P^{o}(2 p) \rightarrow{ }^{2} P^{o}(3 p)$ & ${ }^{2} P^{o}(2 p) \rightarrow{ }^{2} S(4 s)$ & ${ }^{2} S(3 s) \rightarrow{ }^{2} P^{o}(3 p)$ & ${ }^{2} S(3 s) \rightarrow{ }^{2} S(4 s)$ & ${ }^{2} P^{o}(3 p) \rightarrow{ }^{2} S(4 s)$ \\
\hline \multirow[t]{6}{*}{${ }^{11} \mathrm{~B}$} & 1000 & 40029.504 & 48616.840 & 55011.926 & 8587.336 & 14982.422 & 6395.086 \\
\hline & 2000 & 40045.740 & 48619.119 & 55016.792 & 8573.378 & 14971.051 & 6397.673 \\
\hline & 3000 & 40048.584 & 48619.623 & 55017.802 & 8571.039 & 14969.218 & 6398.179 \\
\hline & 4000 & 40049.469 & 48619.749 & 55018.160 & 8570.280 & 14968.691 & 6398.411 \\
\hline & 5000 & 40049.847 & 48619.802 & 55018.331 & 8569.956 & 14968.484 & 6398.529 \\
\hline & $5100^{\mathrm{a}}$ & $40049.887(200)$ & $48619.806(50)$ & $55018.349(100)$ & $8569.920(200)$ & $14968.462(100)$ & $6398.542(70)$ \\
\hline${ }^{10} \mathrm{~B}$ & $5100^{\mathrm{a}}$ & $40050.054(200)$ & $48619.883(50)$ & $55018.428(100)$ & $8569.828(200)$ & $14968.374(100)$ & $6398.545(70)$ \\
\hline${ }^{\infty} \mathrm{B}$ & $5100^{\mathrm{a}}$ & $40048.200(200)$ & $48619.039(50)$ & $55017.551(100)$ & $8570.839(200)$ & $14969.351(100)$ & $6398.513(70)$ \\
\hline Experiment ${ }^{\mathrm{b}}$ & $5100^{\mathrm{a}}$ & 40039.650 & 48611.817 & 55010.181 & 8572.167 & 14970.531 & 6398.364 \\
\hline
\end{tabular}

\footnotetext{
${ }^{\mathrm{a}}$ This basis set was generated with a more extensive optimization of the nonlinear parameters.

${ }^{\mathrm{b}}$ For transitions frequencies involving $P$ states, there is significant fine-structure splitting (up to several $\mathrm{cm}^{-1}$ in magnitude). In this table, we show the frequencies corresponding to the transitions involving lowest-lying sublevels.
}

our nonrelativistic transition energies are converged to about $0.05-0.20 \mathrm{~cm}^{-1}$.

Let us now examine the agreement of the calculated transition energies with the experimental values. As these calculations have included neither relativistic nor QED corrections, one may expect some deviations between the calculated and the experimental values. They indeed appear, but never exceed about $10 \mathrm{~cm}^{-1}$. It is interesting to examine the transition energies in the series of the following consecutive excitations: ${ }^{2} P^{o}(2 p) \rightarrow{ }^{2} S(3 s),{ }^{2} S(3 s) \rightarrow{ }^{2} P^{o}(3 p)$, and ${ }^{2} P^{o}(3 p) \rightarrow$ ${ }^{2} S(4 s)$. The corresponding calculated ${ }^{11} \mathrm{~B}$ transition energies are 40 049.887(200), 8569.920(200), and 6398.542(70) $\mathrm{cm}^{-1}$, respectively. As those excitations correspond to promoting the valence electron between increasingly higher pairs of adjacent $S$ and $P$ states, one would expect to see the calculated transition energies to become closer to the experimental values. This is because the relativistic contribution is mainly due to the state of the core electrons and, as the valence electron becomes more removed from the atom (which happens when it gets excited to increasingly higher states), the state of the core is affected increasingly less. This means that the relativistic contribution to the transition energy should decrease for higher $S$ - $P$ and $P-S$ transitions. With this, the calculated transition energies should become closer to the experimental transitions, and this indeed is what happens in the series of the three mentioned transitions. The differences between the calculated and the experimental transition energy values are 10.237, 7.753, and $0.178 \mathrm{~cm}^{-1}$, respectively. As one sees, the transition energy for the highest transition is very close to the experimental value.

As the goal of this work has been to provide more accurate calculated reference values for boron, aside from the energy we also calculated some other expectation values that provide some additional characterization of the wave functions of the considered states. The expectations values are shown in Table III. They have been calculated using the basis sets of 5100 ECGFs. Let us first comment on the expectation value of the nucleus-electron distance $\left\langle r_{i}\right\rangle$. It characterizes the spatial extent of the wave function. As expected, as the level of excitation increases, $\left\langle r_{i}\right\rangle$ gets bigger. For the highest ${ }^{2} S\left(1 s^{2} 2 s^{2} 4 s\right)$ state, it is almost three times larger than for the ground ${ }^{2} P\left(1 s^{2} 2 s^{2} 2 p\right)$ state. Also, $\left\langle r_{i}\right\rangle$ shows that, when the nuclear mass increases, the electron density contracts. This contraction is smaller for the ground state than for the higher states. However, interestingly, the contraction does not increase monotonically with the level of excitation. It is slightly larger for the ${ }^{2} S\left(1 s^{2} 2 s^{2} 3 s\right)$ state than for the ${ }^{2} P\left(1 s^{2} 2 s^{2} 3 p\right)$ state, even though the latter has higher energy.

TABLE III. Expectation values of powers of the inter-particle distances and contact densities. All values are in a.u. The results obtained with 5100 function basis sets are shown. The estimated uncertainties due to finite size of the basis are given in parentheses.

\begin{tabular}{|c|c|c|c|c|c|c|c|c|c|}
\hline State & Isotope & $\left\langle 1 / r_{i}\right\rangle$ & $\left\langle 1 / r_{i j}\right\rangle$ & $\left\langle r_{i}\right\rangle$ & $\left\langle r_{i j}\right\rangle$ & $\left\langle r_{i}^{2}\right\rangle$ & $\left\langle r_{i j}^{2}\right\rangle$ & $\left\langle\delta\left(\mathbf{r}_{i}\right)\right\rangle$ & $\left\langle\delta\left(\mathbf{r}_{i j}\right)\right\rangle$ \\
\hline \multirow[t]{3}{*}{${ }^{2} P^{o}\left(1 s^{2} 2 s^{2} 2 p\right)$} & ${ }^{10} \mathrm{~B}$ & $2.27887027(5)$ & $0.7666756(4)$ & $1.348148(2)$ & $2.245196(5)$ & $3.10186(2)$ & $6.70726(5)$ & $14.3659(30)$ & $0.35399(5)$ \\
\hline & ${ }^{11} \mathrm{~B}$ & $2.27888137(5)$ & $0.7666786(4)$ & $1.348143(2)$ & $2.245188(5)$ & $3.10184(2)$ & $6.70721(5)$ & $14.3661(30)$ & $0.35399(5)$ \\
\hline & ${ }^{\infty} \mathrm{B}$ & $2.27899296(5)$ & $0.7667092(4)$ & $1.348088(2)$ & $2.245104(5)$ & $3.10160(2)$ & $56.70672(5)$ & $14.3683(30)$ & $0.35404(5)$ \\
\hline \multirow[t]{3}{*}{${ }^{2} S\left(1 s^{2} 2 s^{2} 3 s\right)$} & ${ }^{10} \mathrm{~B}$ & $2.22598184(20)$ & $0.6709509(3)$ & $2.103834(4)$ & $3.660657(8)$ & $10.67368(3)$ & $21.77187(6)$ & $14.5047(30)$ & $0.35829(9)$ \\
\hline & ${ }^{11} \mathrm{~B}$ & $2.22599301(20)$ & $0.6709539(3)$ & $2.103824(4)$ & $3.660639(8)$ & $10.67357(3)$ & $21.77164(6)$ & $14.5049(30)$ & $0.35829(9)$ \\
\hline & ${ }^{\infty} \mathrm{B}$ & $2.22610521(20)$ & $0.6709844(3)$ & $2.103715(4)$ & $3.660453(8)$ & $10.67244(3)$ & $21.76939(6)$ & $14.5071(30)$ & $0.35834(9)$ \\
\hline \multirow[t]{3}{*}{${ }^{2} P^{o}\left(1 s^{2} 2 s^{2} 3 p\right)$} & ${ }^{10} \mathrm{~B}$ & $2.21717677(30)$ & $0.6567476(4)$ & $2.483814(15)$ & $4.404871(30)$ & $17.06735(10)$ & $34.54484(20)$ & $14.4788(10)$ & $0.35755(5)$ \\
\hline & ${ }^{11} \mathrm{~B}$ & $2.21718783(30)$ & $0.6567505(4)$ & $2.483806(15)$ & $4.404856(30)$ & $17.06724(10)$ & $34.54463(20)$ & $14.4790(10)$ & $0.35755(5)$ \\
\hline & ${ }^{\infty} \mathrm{B}$ & $2.21729894(30)$ & $0.6567791(4)$ & $2.483719(15)$ & $4.404712(30)$ & $17.06621(10)$ & $34.54257(20)$ & $14.4812(10)$ & $0.35760(5)$ \\
\hline \multirow[t]{3}{*}{${ }^{2} S\left(1 s^{2} 2 s^{2} 4 s\right)$} & ${ }^{10} \mathrm{~B}$ & $2.20623420(80)$ & $0.6352221(15)$ & $3.598680(50)$ & $6.611520(100)$ & $44.84099(100)$ & $89.99769(300)$ & $14.4906(30)$ & $0.35790(7)$ \\
\hline & ${ }^{11} \mathrm{~B}$ & $2.20624518(80)$ & $0.6352247(15)$ & $3.598666(50)$ & $6.611495(100)$ & $44.84061(100)$ & $89.99694(300)$ & $14.4908(30)$ & $0.35790(7)$ \\
\hline & ${ }^{\infty} \mathrm{B}$ & $2.20635557(80)$ & $0.6352516(15)$ & $3.598524(50)$ & $6.611241(100)$ & $44.83685(100)$ & $89.98943(300)$ & $14.4930(30)$ & $0.35795(7)$ \\
\hline
\end{tabular}


Another property worth noting is the electron density at the nucleus [i.e., the $\left\langle\delta\left(\mathbf{r}_{i}\right)\right\rangle$ expectation value]. With the increasing level of excitation this density increases, which is understandable because, as the valence electron moves further away from the nucleus, the core electrons contract leading to their higher density at the nucleus. Also, for all the states, the higher nuclear mass leads to a slightly higher $\left\langle\delta\left(\mathbf{r}_{i}\right)\right\rangle$.

\section{SUMMARY}

In this work, we obtained the four lowest ${ }^{2} S$ and ${ }^{2} P$ states of the two stable isotopes of the boron atom ${ }^{10} \mathrm{~B}$ and ${ }^{11} \mathrm{~B}$. High accuracy has been achieved by employing large basis sets of explicitly correlated Gaussian functions and optimizing their nonlinear parameters with an approach that utilizes the analytical gradient of the energy determined with respect to those parameters. The results demonstrate that five-electron atomic systems can now be calculated with a comparable accuracy as for atoms with four electrons. The lack of accounting for relativistic and QED effects results in some discrepancies between the calculated and the experimental transition energies. Thus, the next task in our work will be to develop algorithms for computing the relativistic corrections.
[1] C. Schwartz, Int. J. Mod. Phys. E 15, 877 (2006).

[2] C. Schwartz (2006), e-print arXiv:math-ph/0605018.

[3] H. Nakashima and H. Nakatsuji, J. Chem. Phys. 127, 224104 (2007).

[4] M. Puchalski and K. Pachucki, Phys. Rev. A 73, 022503 (2006).

[5] Z.-C. Yan, W. Nörtershäuser, and G. W. F. Drake, Phys. Rev. Lett. 100, 243002 (2008).

[6] M. Puchalski, D. Kedziera, and K. Pachucki, Phys. Rev. A 80, 032521 (2009).

[7] T. Kato, Commun. Pure Appl. Math. 12, 151 (1957).

[8] D. M. Fromm and R. N. Hill, Phys. Rev. A 36, 1013 (1987).

[9] T. K. Rebane, V. S. Zotev, and O. N. Yusupov, Zh. Eksp. Teor. Fiz. 110, 55 (1996) [Sov. Phys. JETP 83, 28 (1996)].

[10] V. S. Zotev and T. K. Rebane, Phys. Rev. A 65, 062501 (2002).

[11] M. Puchalski and K. Pachucki, Phys. Rev. A 81, 052505 (2010).

[12] K. Pachucki and J. Komasa, Phys. Rev. Lett. 92, 213001 (2004).

[13] K. Pachucki and J. Komasa, Phys. Rev. A 73, 052502 (2006).

[14] M. Stanke, D. Kedziera, S. Bubin, and L. Adamowicz, Phys. Rev. Lett. 99, 043001 (2007).

[15] M. Stanke, J. Komasa, S. Bubin, and L. Adamowicz, Phys. Rev. A 80, 022514 (2009).

[16] S. Bubin, J. Komasa, M. Stanke, and L. Adamowicz, J. Chem. Phys. 131, 234112 (2009).
[17] S. Bubin, J. Komasa, M. Stanke, and L. Adamowicz, Phys. Rev. A 81, 052504 (2010).

[18] S. Bubin, J. Komasa, M. Stanke, and L. Adamowicz, J. Chem. Phys. 132, 114109 (2010).

[19] M. Cafiero, S. Bubin, and L. Adamowicz, Phys. Chem. Chem. Phys. 5, 1491 (2003).

[20] S. Bubin, M. Cafiero, and L. Adamowicz, Adv. Chem. Phys. 131, 377 (2005).

[21] S. Bubin and L. Adamowicz, J. Chem. Phys. 124, 224317 (2006).

[22] S. Bubin and L. Adamowicz, J. Chem. Phys. 128, 114107 (2008).

[23] M. Hamermesh, Group Theory and Its Application to Physical Problems (Addison-Wesley, Reading, 1962).

[24] Yu. Ralchenko, A. Kramida, J. Reader, and NIST ASD Team (2010), NIST Atomic Spectra Database, version 4.0 (online), available at [http://physics.nist.gov/asd].

[25] S. Bubin, M. Stanke, and L. Adamowicz, J. Chem. Phys. 131, 044128 (2009).

[26] V. I. Kukulin and V. M. Krasnopol'sky, J. Phys. G: Nucl. Phys. 3, 795 (1977).

[27] K. Varga and Y. Suzuki, Phys. Rev. C 52, 2885 (1995).

[28] Y. Suzuki and K. Varga, Stochastic Variational Approach to Quantum-Mechanical Few-Body Problems (Springer, Berlin, 1998). 